\title{
PROTAGONISMO Y MILITANCIA: EL ESTILO DE GESTIÓN DEL MOVIMIENTO BARRIOS DE PIE EN EL MINISTERIO NACIONAL DE DESARROLLO SOCIAL, ARGENTINA 2004 - 2009
}

LEADERSHIP AND MILITANCE: BARRIOS DE PIE MOVEMENT'S MANAGEMENT STYLE IN SOCIAL MINSTRY OF NACIONAL STATE, ARGENTINA 2004- 2009.

\section{AGUSTINA GRADIN*}

\section{R E S U M E N}

En Argentina, durante los gobiernos de Néstor y Cristina Kirchner (2003-2015), los movimientos sociales fueron actores claves del proceso de reconstrucción del Estado nacional, luego de la crisis política, social y económica de 2001-2002. Por esto, se propone analizar la experiencia del Movimiento de desocupados Barrios de Pie en el Ministerio de Desarrollo Social de la Argentina durante el periodo 2004-2009, en donde ocupó diferentes espacios de gestión estatal. La incorporación del militante como actor estratégico de la gestión promovió un estilo basado en aspectos subjetivos como la voluntad política, el compromiso y el protagonismo, e implicó una revalorización de la militancia política, social y comunitaria como elemento estratégico de la política pública.

PALABRAS CLAVE: Estilo de gestión, protagonismo, militancia, comunitarismo, organizaciones de desocupados, Movimiento Barrios de Pie.

\section{A B S T R A C T}

In Argentina, during the governments of Néstor and Cristina Kirchner (2003 - 2015), social movements were key actors in the process of reconstruction of the national State, after the political, social and economic crisis of 2001 - 2002. Therefore, we propose analyze the experience of the Movement of unemployed Barrios de Pie in the Ministry of Social Development of Argentina during the period 2004 - 2009, where he occupied different areas of state management. The incorporation of the militant as a strategic actor of the management promoted a style based on subjective aspects such as political will, commitment and protagonism, and implied a revaluation of the political, social and community militancy as a strategic element of the public policy.

KEYWORDS: Management style, leadership, militancy, communitarianism, unemployed's organization, Barrios de Pie Movement.

\footnotetext{
"Doctora en Ciencias Sociales de la Universidad de Buenos Aires. CONICET/FLACSO, Argentina. Correo electrónico: agradin@flacso.org.ar
} 


\section{INTRODUCCIÓN}

En la Argentina, para superar la crisis económica, política y social que atravesó el modelo neoliberal en 2001 2002, fue necesario el fortalecimiento y la ampliación del Estado nacional para que guiara el proceso de desarrollo productivo e inclusivo encabezado por el gobierno de Néstor Kirchner (2003-2007). Este proceso involucró la participación política de nuevas organizaciones sociopolíticas, entre las cuales se destacaron las organizaciones de desocupados, actores claves de la resistencia al modelo neoliberal. A éstas se les propuso, por primera vez, incorporarse a la gestión pública para diseñar y ejecutar soluciones a sus demandas. Este nuevo rol asumido por algunas organizaciones, entre los cuales se encuentra el movimiento Barrios de Pie (en adelante BDP), redefinió sus formas de acción y sus prácticas dentro y fuera del Estado. Esto impactó en las mediaciones entre el Estado y la sociedad, al instituirlos como un nuevo canal de vinculación entre ambos. Este trabajo se propone analizar la experiencia de BDP en el Ministerio de Desarrollo Social de la nación durante el periodo 2004-2009, recuperando un aspecto desarrollado en la tesis doctoral titulada "La participación política de las organizaciones de desocupados en el Estado nacional durante el periodo 2003-2009. La experiencia del Movimiento Barrios de pie: sus límites y potencialidades" (Gradin, 2016). El trabajo analiza sus prácticas en el Estado a partir del concepto de estilo gestión ya que se considera que permite comprender aspectos sustantivos del trabajo político en el Estado de las organizaciones de desocupados en general y particularmente de BDP. La incorporación del militante como actor estratégico de la gestión promovió un estilo basado en aspectos subjetivos como la voluntad política, el compromiso y el protagonismo, como intentaremos mostrar en este artículo.

Por lo tanto, en el siguiente apartado se desarrolla el entramado teórico metodológico a partir del cual se construye el análisis. En el tercer apartado, se presenta los principales hallazgos del trabajo de campo realizado, y en el cuarto apartado, aborda los principales argumentos de nuestro análisis. Por último, en las reflexiones finales se propone un epílogo de la experiencia. 


\section{EL ESTILO DE GESTIÓN DE BDP: COORDENADAS TEÓRICO - METODOLÓ- GICAS}

En el escenario estatal, poroso en sus fronteras con la sociedad y en permanente construcción y disputa, las políticas estatales se comprenden como nudos que permiten analizar el proceso social. Estos nudos, a los fines de nuestros objetivos de investigación, son analizados como dispositivos (Tiranti, 2013; Gradin, 2013; Dallorso, 2013; Gradin y Tiranti, 2012) con dimensiones, características y propiedades específicas. En un dispositivo se inscriben una multiplicidad de elementos del orden de los saberes y discursos; las tecnologías, sus mecanismos, procedimientos, normas y rutinas, diferentes actores (estatales y no estatales), con sus experiencias, prácticas, expectativas y relaciones de poder. Todos estos elementos se vincu-

\footnotetext{
1 BDP surge como organización de desocupados a fines del año 2001, cuando un sector de la central de Trabajadores Argentinos, autodenominado CTA de los Barrios, se separó de la federación de Tierra y Vivienda (FTV), debido a diferencia políticas sobre la coyuntura de la crisis político-social de 2001 y el gobierno interino de Eduardo Duhalde (2002 - 2003). En el año 2003, luego de la asunción de Néstor Kirchner como presidente (2003 -2007), BDP comienza un proceso de acercamiento al gobierno que deviene en su participación de diversas políticas estatales implementadas durante el periodo analizado. En las elecciones legislativas de medio término del 2009 durante el primer gobierno de
}

lan entre sí con la finalidad de conseguir un efecto, en este caso, la implementación de una política en el territorio o de un programa nacional. Es en la forma de funcionamiento de los dispositivos, la relaciones entre los diferentes elementos donde, se considera, se puede visualizar los estilos de gestión de cada actor particular (Mancini, 2011; Schavelzon, 2007; Zaremberg, 2004; Mazzuca, 2002).

El involucramiento de las organizaciones de desocupados en la gestión de políticas condicionó el funcionamiento de los dispositivos de gestión. Por lo tanto, se considera que en el funcionamiento de los dispositivos emergieron estilos de gestión propios, con dimensiones, características y propiedades particulares. Del análisis del funcionamiento de los dispositivos analizados en este trabajo, se podrá iluminar el estilo de gestión desplegadas por BDP en su participación política de los espacios de gestión del Ministerio de Desarrollo social de la Nación durante el periodo $2004-2009^{1}$.

Cristina Fernández (2007 - 2011), BPD termina de distanciarse políticamente del oficialismo, asumiéndose directamente como un actor de la oposición en el proceso de selección de las autoridades legislativas de la cámara baja del Congreso nacional luego de dicha elección. Este hecho fue conocido como la conformación del Grupo "A", que contenía a la mayoría de la oposición en el congreso nacional, que intentó un acuerdo político que le permitiera vencer al Kirchnerismo dentro de la cámara de Diputados. BDP, a través de Libres del Sur, fue parte de este proceso. Esto significó el alejamiento definitivo 
El estudio se propuso analizar la relación entre BDP y el Estado nacional en términos de participación política porque se considera que condensa, tanto una mirada estratégica de la acción de estas organizaciones, así como contiene elementos simbólicos e identitarios de la acción colectiva que también deben ser considerados en el análisis para comprender el fenómeno estudiado $^{2}$. A su vez, esta conceptualización implica una mirada superadora de la concepción técnica de la participación como herramienta de legitimación de políticas estatales, e incorpora y pone en juego la dimensión del poder que atraviesa la relación entre el Estado y las organizaciones de desocupados. Y en esta relación, si bien se reconocen las desigualdades existentes en términos de recursos y capacidades de cada actor, ambos son concebidos como sujetos de la misma. Sujetos que portan y construyen una forma de ver el mundo, de habitarlo, de soñarlo y que actúan con una racionalidad limitada y con arreglo a valores y a fines, según el momento histórico. En este sentido, la participación es política en tanto que implica la construcción de relaciones de poder entre los dos principales actores, las organizaciones de desocupados y el Estado nacional. Por lo tanto, el interés es la participación política de BDP "en" el Estado nacional y a través de las políticas estatales, porque se considera que fueron un espacio fundamental para la reconstrucción de la relación entre el Estado y la sociedad durante el periodo analizado.

En función de estos supuestos teóricos, este trabajo de investigación fue abordado desde un enfoque metodológico cualitativo y desde una perspectiva sociopolítica e histórica, orientado al análisis de la participación política de BDP en el Estado nacional durante el periodo 2004-2009 en Argentina. Para esto, se proponen el concepto de estilo de gestión estatal como clave conceptual analizar la experiencia de BDP en el escenario estatal, específicamente en el Ministerio de Desarrollo Social de la Nación. Desde el 2004 y hasta su alejamiento del gobierno en 2009, BDP ocupó diferentes cargos en del espacio kirchnerista al cual había pertenecido desde su incorporación al gobierno y a través de diferentes armados electorales (Gradin, 2016).

2 De la matriz estratégica se recupera el énfasis en la vinculación con el sistema político y las disputas por el poder dentro de una sociedad. De las perspectivas identitarias se recuepra la preocupación por la construcción subjetiva de las formas de organización y de acción, aún en íntima relación con otras tradiciones organizacionales. A su vez, estas dimensiones analíticas, la acción estratégica y la construcción identitaria de las organizaciones de desocupados, se inscriben necesariamente en los procesos históricos estructurales vinculados principalmente a la producción y apropiación de la riqueza socialmente producida, y a la construcción de consensos y disputas alrededor de dicho proceso. 
el Ministerio, así como promovió e implementó una batería de programas de promoción social en el territorio. Para este trabajo se analizó específicamente la experiencia de la Dirección Nacional de Asistencia Comunitaria entre los años 2004-2006, y de la Subsecretaría de Organización y Capacitación popular y sus iniciativas del 2006 al 2009. Para analizar estas experiencias el estudio se centró en los saberes $\mathrm{y}$ valores propios de quienes participan de la misma, sus lógicas, racionalidades y prácticas que pusieron en práctica en su paso por el Estado, su forma de vinculación con la sociedad civil, las estructuras de autoridad y las formas de circulación de la información al interior de la burocracia, la distribución de atribuciones, competencias y responsabilidades al interior de las diferentes áreas del Estado, los criterios técnico administrativos que las organizaron y controlaron, y por último, los recursos materiales, su procedencia y formas de distribución. Del análisis de estas dimensiones establecimos el estilo de gestión estatal de BDP durante el periodo analizado.

El trabajo de campo se realizó durante el periodo 2006-2012 en diferentes etapas. Se realizaron un total de 25 entrevistas, de las cuales 12 fueron a funcionarios y trabajadores vinculados a BDP, 3 a trabajadores y funcionarios técnicos del Ministerio no militantes de dicha agrupación, y 10 fueron hechas a militantes territoriales. Las observaciones directas se realizaron durante los años 2007 - 2008, como participante del Programa de Promotores Territoriales para el cambio social del Ministerio de Desarrollo social de la Nación, y se enfocó en la observación de los espacios de gestión estatal dirigidos por BDP en dicho ministerio. La información brindada por los entrevistados fue contrastada y complementada por fuentes secundarias de información como documentos oficiales e institucionales, noticias de la prensa escrita, informes de gestión, evaluaciones, etc.

\section{EL ESTILO DE GESTIÓN ESTATAL: LA EXPERIEN- CIA DE BDP EN EL MINIS- TERIO DE DESARROLLO SOCIAL DE LA NACIÓN}

La participación de BDP en el Estado nacional, en un principio anclada en el territorio y en la gestión de políticas de transferencia condicionada de ingresos, da un salto político cuando a fines de 2003 BDP comienza a acercarse al espacio político kirchnerista (Gradin, 2016). En este apartado se desarrlló la experiencia de BDP en la gestión pública del Estado nacional, específicamente en el Ministerio de Desarrollo Social de la Nación. Para 
analizar el estilo de gestión estatal que emergió de la experiencia de BDP en los espacios de gestión se utilizó la información surgida del trabajo de campo. El argumento que guía este artículo propone que el estilo de gestión construido por BDP en su experiencia en el Ministerio, implicó una revalorización de la militancia política, social y comunitaria como elemento estratégico de la modalidad de implementación de las mismas. Ahora bien, la experiencia de gestión de este actor, su capacidad de acción, su racionalidad militante y su experiencia organizativa, se encontraron con la racionalidad burocrática, la capacidad técnica y la experiencia de gestión del Ministerio, dejando emerger una síntesis entre ambos estilos de gestión. Este estilo de gestión, basado en el protagonismo de los militantes en el quehacer diario y sintetizado en la popular frase “disputar el Estado", se arraiga en una experiencia cuyos matices y contradicciones iluminan, a nuestro entender, aspectos de la participación política de las organizaciones de desocupados en el Estado nacional ${ }^{3}$.

De esto nos encargaremos en los siguientes apartados.

\footnotetext{
${ }^{3}$ En el transcurso de este texto, se señalarán entre comillas y con letra Itálica las expresiones correspondientes a categorías nativas.

${ }^{4}$ En la Resolución conjunta N³5/2004 del Ministerio de Desarrollo Social de la Nación y de la Secretaría de la Gestión Pública de la Presidencia de la Nación, con fecha del 4 de junio de
}

\section{1. Del territorio al organigrama del Ministerio de Desarrollo So- cial de la nación}

La relación de BDP con las políticas sociales tuvo un giro, en términos de Massetti (2011), cuando a partir del 2003 se incorporaron al diseño de las políticas sociales, estrategias de fortalecimiento de capital social, la economía social y el desarrollo local, dando un salto en el tipo de programas que se implementaban. Se combinaron los programas masivos de transferencia condicionada de ingresos que analizamos en el capítulo anterior, con programas de promoción social, productivos, y/ o educativos.

La estrategia de participación en las políticas estatales, sufrió otro giro cuando fueron convocadas a "sumarse" a la estructura de gestión del propio Ministerio de Desarrollo social de la Nación. A mediados del año 2004, el coordinador nacional de BDP, Jorge Ceballos, fue convocado por Alicia Kirchner, Ministra de Desarrollo Social de Nación, para ocupar el cargo de Director Nacional de Asistencia Comunitaria (en adelante DAC) de dicho Ministerio ${ }^{4}$.

2004, se estableció la reorganización, derogación y creación de nuevas unidades ejecutoras dentro de la estructura ministerial, de acuerdo al proceso de transformación del rol del Estado, y a los objetivos y líneas estratégicas definidos por el Ministerio. En este marco, entre otras uni- 
El 30 de junio de 2006, Ceballos fue nombrado Subsecretario de Organización y Capacitación Popular del Ministerio. Desde sus comienzos, la subsecretaría adoptó un formato que reproducía la lógica de organización de BDP, incorporando a la gestión pública dos cuestiones fundamentales, la organización y el comunitarismo como formas de gestión, que BDP los sintetizó en el concepto de "protagonismo" como veremos más adelante. El protagonismo, entendido como una estrategia colectiva de resolución de problemas, era parte de sus saberes y de sus prácticas en la gestión territorial de políticas estatales, como vimos en el capítulo anterior. Esto permite suponer que la organización puso a disposición de la gestión pública, su bagaje de experiencias y saberes construidos en la gestión social de su organización en el territorio.

La subsecretaría impulsó un abanico de programas nacionales relacionados a una problemática diferente. Entre ellos, se destacaron el Programa de Alfabetización en Salud, orientado a la

dades, se crearon la Secretaría de Gestión y articulación cuyo objetivo general era la construcción del modelo de gestión social estratégico e integral sustentado en interacción y articulación con otros organismos del Estado y las organizaciones de la sociedad civil, desde una mirada territorial y transversal de las problemáticas sociales. Dependiendo de dicha Secretaría, se creó la Dirección Nacional de Asistencia Comunitaria, para la cual se incorporó Jorge Ceballos como Director Nacional.

${ }^{5}$ Durante todo el periodo analizado BDP a través de su armado político partidario Libres del formación de Promotores en Salud Comunitaria; el Programa Contálo Vos, de promoción y fortalecimiento de iniciativas de comunicación Comunitaria; el Programa Haciendo Historia, orientado a organizaciones de la sociedad civil, y cuyos objetivos eran promover autonomía, capacidad de gestión y de incidencia en las políticas públicas; y el Programa Vínculos, experiencias comunitarias en Educación popular, que financiaba proyectos que desarrollaban prácticas comunitarias. En cada uno de estos programas se incorporaron militantes de BDP como personal profesional y técnico del MDS. Estos programas recuperaban las experiencias de trabajo social desplegadas en el territorio por BDP antes de asumir la gestión pública (Gradin, 2016).

A fines de 2008, a raíz de una serie de diferencias planteadas por BDP en diferentes comunicados de prensa de la organización, se retiró de sus espacios de gestión en el Ministerio de Desarrollo Social de la Nación y de otros ministerios ${ }^{5}$.

Sur, participó de lo que se conoció como la "transversalidad kirchnerista". Un espacio amplio de centro izquierda donde confluían organizaciones de diferentes tradiciones políticas, y que encontraba en Néstor Kirchner su referente natural. Durante la primera presidencia de Cristina Fernández, y luego de la derrota política en la disputa por las retenciones móviles al campo, el Kirchnerismo decide recostarse en el Partido Justicialista para recuperar la capacidad política, restándole apoyo a BDP. La organización decide primero abandonar sus espacios en el 
3.2. La experiencia de los espacios de gestión

Tanto en la experiencia de la DAC como de la Subsecretaría, se presentaron elementos comunes que tiene relación principalmente con las características de los espacios de gestión propiamente supra estructurales. Es decir, espacios de gestión centralizados que contienen diferentes líneas de acción en relación a su misión y objetivos. En ambas experiencias se conformaron gabinetes de gestión alrededor del director o del secretario, compuestos por militantes de BDP y principalmente vinculados al trabajo territorial pero también al frente juvenil.

Cuando Ceballos asumió la DAC en 2004, conformó un equipo de 4 militantes que lo acompañó toda la gestión y que luego pasaron a formar parte de la Subsecretaria. Ninguno tenía experiencia de gestión pública previa, y casi ninguno contaba con estudios universitarios ni formación profesional. Si acreditaban una extensa experiencia política y social. Según las entrevistas realizadas, los primeros meses de gestión fueron muy difíciles porque significaron conocer e incorporar una cantidad importante de "formas de hacer y de pensar" vinculadas a la práctica burocrática del ministerio. La DAC contenía en su seno varios programas y direcciones, que a su vez respondían a otros espacios políticos, por lo que el primer paso fue entablar relaciones políticas con éstas para poder aceitar el funcionamiento general de la dirección. En el mismo sentido, se entablaron relaciones con otras dependencias a los fines de coordinar y facilitar acciones en conjunto.

La forma de organización de la DAC fue colectiva, en tanto que, si bien Ceballos era el referente y responsable de la dirección, el equipo funcionaba, según sus propios protagonistas, colectivamente y corresponsablemente. Es decir, se mantenía la democracia interna y la horizontalidad en la toma de decisiones que se utilizaba en la organización. Con el correr del tiempo se fueron incorporando otros militantes en funciones técnicas o administrativas de la dirección, así como en otras dependencias del Ministerio, gracias a acuerdos políticos generados. El criterio de selección de los militantes que ingresaban a la gestión, ya sea como trabajadores rentados o ad honorem respondió, según lo indicado por varios de los entrevistados, a la necesidad política de garantizar el funcionamiento de los espacios de gestión, a la necesidad de "hacer crecer la organización a partir de la implementación

Estado Nacional, y luego establecer un nuevo marco de alianzas políticas, pasando directamente al espacio opositor. 
de los recursos del Ministerio" (Entrevista 1), y a la necesidad de fortalecer la organización para la disputa política. $Y$ esto se relacionaba claramente a su forma de asumir la gestión. Como señala un entrevistado:

"En el Ministerio nos conocían como los piqueteros, pero cada vez que venía alguien a pedir algo, y en ese momento eran muchos los que se acercaban, los mandaban a hablar con nosotros. Siempre, los policías de la entrada hasta las trabajadoras sociales de planta baja, cuando no podían hacer nada, nos los mandaban a nosotros.... ¿Por qué? Porque sabían que nosotros íbamos a agotar todas las posibilidades para ver si podíamos resolverlos... hemos estado hasta las 12 de la noche con personas dando vuelta por las diferentes dependencias, tocando puertas, tratando de gestionar soluciones" (Entrevista 5).

Esta práctica implicaba una sensibilidad especial con respecto de los problemas sociales y de las personas que se acercaban en busca de soluciones, corriendo la centralidad del apego a las normas a las características subjetivas del militante como actor central del proceso de gestión. Este compromiso con la gestión implicaba, también, que los militantes no pudieran contar con el tiempo para poder militar en sus frentes de masas como antes de incorporarse a la gestión. Con el tiempo, "la gestión" se convirtió en un frente de masas en sí mismo, en términos de construcción política de la organización.

La subsecretaría, a diferencia de la DAC, además de contar con un rango más elevado en el organigrama del Ministerio, contaba con la posibilidad de crear y ejecutar programas propios, además de continuar implementando otros programas que se venían ejecutando. Para BDP la subsecretaría debía seguir tres objetivos internos "permitir el crecimiento y fortalecimiento de la propia organización, ampliar el abanico de alianzas políticas, y crecer en experiencia de gestión pública que le permita demostrar capacidad y que eso tenga un correlato en la política" (Entrevista 17). Para garantizar el funcionamiento de la subsecretaría, y la "llegada" a diferentes territorios y organizaciones, se replicó el esquema de gestión colectiva de la DAC. Se organizó un gabinete donde participaban los responsables políticos de cada dirección nacional y de cada dirección de línea, así como los responsables de los diferentes programas. A su vez, cada dirección tenía su propio equipo 
de asesores y técnicos que se encargaban de desarrollar las políticas definidas por el gabinete.

La relación con el territorio y las organizaciones era filtrada orgánicamente por BDP, a través de sus militantes. Para cumplir con sus objetivos políticos, la organización focalizó sus esfuerzos y militantes en la Dirección Nacional de Enlace entre Organizaciones Públicas y el Tercer Sector (DNE), que tenía por objetivo promover la creación redes horizontales de participación comunitaria entre el sector público y las organizaciones de la sociedad civil. De ésta dependían la Dirección de Prácticas en Oficios Populares (DIPOP) y la Dirección de Educación Social y Popular (DESP), desde donde la organización generaba iniciativas políticas propias.

La DIPOP impulsaba el apoyo técnico y financiero a proyectos de formación y capacitación en oficios ejecutados por organizaciones de la sociedad civil. La DESP, en un sentido similar, respaldaba proceso de capacitación, asistencia técnica, educación popular y formación de líderes comunitarios que promuevan la organización y la integración, estimulando la participación de la sociedad. Para cumplimentar este objetivo, la DESP implementaba un abanico de programas que fueron

${ }^{6}$ Para el año 2006, se informa en Memoria detallada del Estado de la Nación, que 17000 personas pertenecientes a 2.229 organizaciones la cara visible de la subsecretaría y su "caballito de batalla" para lo que ellos mismos definían como "disputar el territorio y el propio Estado". Todos los programas tenían por objetivo promover el "protagonismo" de las organizaciones de la sociedad civil a través de la capacitación y la asistencia técnica y financiera, y fueron pensados, diseñados y ejecutados por $\mathrm{BDP}^{6}$. Todas las iniciativas tenían la misma forma de implementación a través del desarrollo de proyectos sociales en el territorio. Para esto era necesario "gestionar un subsidio". El principal inconveniente con que se encontró la organización fue la dificultad de adecuar la normativa y los procedimientos a las realidades heterogéneas de las organizaciones de base. La escasa institucionalidad de las organizaciones a las cuales estaban dirigidos dificultaba la posibilidad legal de incorporarlas como organismo ejecutor de algún programa específico, y, por lo tanto, de transferirles recursos materiales y financieros que les permita fortalecer su trabajo.

En este sentido, según las entrevistas realizadas, se buscaron alternativas dentro del marco legal del Ministerio para poder contener a todas las organizaciones, aunque no contaran con

participaron en encuentros de capacitación sobre prácticas de salud, educación, derechos humanos, ciudadanía, entre otros. 
los requisitos administrativos necesarios. Una forma fue el formato de concurso nacional como el "Contálo Vos", que por su carácter de legal permitía que se presentaran todas las organizaciones sociales que quisiesen, cuenten o no con personería jurídica ${ }^{7}$. Otro formato utilizado para el desarrollo de actividades fueron los "eventos", los cuales eran gestionados sin necesidad de que interviniese una asociación civil o un tercero, y permitían la adquisición de equipamiento para la actividad específica, que luego era " $d o-$ nada" a la organización que participaba del mismo ${ }^{8}$. Este instrumento permitía financiar cualquier actividad, aunque no se encuadre en un programa específico.

Dicho esto, se debe señalar que la autonomía operativa de BDP en los programas mencionados se caracterizaba principalmente por encontrarse constreñida por las cuestiones normativas y procedimentales de la gestión interna del Ministerio. Aun contando con la autonomía política para implementar los programas que querían, los requisitos legales para la gestión de un proyecto o de un subsidio, así como los "tiempos" requeridos, eran los principales inconvenientes señalados. Estas cuestiones podían "enfriar" la relación con una organización, y “complicar" el proceso de gestión general de la subsecretaria. En este sentido, BDP intentaba acomodar las cuestiones legales y procedimentales a sus propias prácticas, "tratando de trasgredir lo menos posible" (Entrevista 11).

Sumados al expediente y el evento, BDP se apoyó principalmente en el formato del programa "enlatado", simple para facilitar la gestión e implementación del mismo, los manuales y materiales de formación tipo cuadernillos, y los instructivos de presentación de proyectos. En este sentido, es importante señalar que estos formatos "enlatados” permitían organizar y canalizar la demanda. Esto facilitaba la gestión de los proyectos, pero limitaba la capacidad de acción de las organizaciones y de BDP. Al contrario, en los programas de la DNE territorial ejecutaba "a demanda" a través de los eventos y de los subsidios, principalmente proyectos de capacitación amplios, y de sumas importantes de dinero, con una mirada política sobre las relaciones con las organizaciones y

\footnotetext{
${ }^{7}$ Según la Memoria detallada del Estado de la Nación 2006, 1655 grupos se inscribieron en el Concurso, de los cuales 48 fueron seleccionados como ganadores en el 2007, representando a las 24 Provincias y a la Ciudad de Buenos Aires.
} 
con los territorios. La DNE implementaba proyectos de capacitación de amplio alcance y a demanda de las propias organizaciones que las solicitaban. En esos proyectos se podía solicitar financiamiento para mejorar la infraestructura y el equipamiento de la misma, fortaleciendo su capacidad de acción, luego de la finalización del proyecto.

Por último, la incorporación de militantes a la estructura de la subsecretaría fue según las entrevistas, "desordenada", y muchas veces sin criterio político, lo cual generó varias tensiones internas. A su vez, se puso en evidencia la falta de experiencia pública y de profesionales dentro de la organización para absorber los espacios que se abrían. Se convocaron profesionales externos a la organización, pero con algún nivel de vinculación con ella, para ocupar los lugares de técnicos, abogados, contadores, etc. En varias entrevistas también aparecía la relación entre el trabajo y la militancia, desde otra perspectiva. Los militantes que se incorporaban como trabajadores de la subsecretaría continuaban participando de sus frentes de militancia. De hecho, la mayoría tenía responsabilidades políticas en los mismos, lo cual fue uno de los criterios que les permitió acceder a esos lugares de trabajo. Esto generaba una fuerte tensión interna con respecto a la dedicación que estos militantes debían "darle" a la subsecretaría. La organización reclamaba a los militantes que no abandonasen sus responsabilidades de construcción social, y la dinámica de la subsecretaría exigía cada vez más tiempo de parte de ellos. Muchos fueron reemplazados en sus responsabilidades políticas en los frentes de masas, $y$ algunos fueron reemplazados en la gestión, tratando de encontrar un equilibrio entre ambas cuestiones. Según un entrevistado, "terminamos militando la gestión hacia adentro de la propia organización" (Entrevista 6). Esto significaba que los militantes debían convencer a sus propios compañeros, sobre la importancia de la gestión de la subsecretaría en el marco de la estrategia política de construcción de la organización. Esto generó en una primera etapa muchos debates internos, que con el correr del tiempo y con los primeros resultados visibles de la gestión, se fueron resolviendo. Efectivamente, cuando los proyectos de capacitación, los eventos, y los subsidios, "comenzaban llegar al barrio", ya sea para uso propio o para la consolidación de relaciones políticas, la militancia territorial comenzó a entender la importancia de la gestión de la Subsecretaría. 
La relación con los trabajadores de planta permanente del Ministerio, según los entrevistados, se estructuraba sobre dos ejes ${ }^{9}$. En primer lugar, sobre la necesidad de la propia organización que desconocía los procedimientos de gestión interna del Ministerio. Es decir, porque los trabajadores de planta tenían el saber técnico necesario para gestionar la subsecretaría. $Y$ en segundo lugar, porque paralelamente a la asunción de BDP en la subsecretaría, el Ministerio impulsó la incorporación de centenares de trabajadores sociales, en su mayoría jóvenes, que renovaron la planta de técnicos y con los cuales fue más fácil trabajar. De hecho, al ser la subsecretaría completamente nueva, los trabajadores sociales y técnicos que se incorporaron a la misma, lo hicieron con mayor libertad y con más entusiasmo, según las entrevistas realizadas. La estrategia de BDP, en este sentido, fue, por un lado, incorporarlos como actores a la gestión cotidiana, "democratizar el Estado", y por otro, defender los derechos del trabajador para con el Ministerio como patronal. Esto construía un escenario complejo para gestionar diariamente, pero era muy valorado por los propios técnicos y profesiona$\operatorname{les}^{10}$. Con respecto a la relación con los trabajadores no militantes de la subsecretaría, los entrevistados señalaron dos cuestiones que atravesaron sus prácticas. En primer lugar, los "prejuicios" que existían tanto de parte de BDP para con la "burocracia" (usado en términos peyorativos), como viceversa. Los prejuicios de los trabajadores para con los militantes de BPD, igualándolos a los "políticos" (también peyorativamente). Estos prejuicios teñían la relación entre ambos actores, pero con la práctica, según los entrevistados, fueron desarmándose. La clave era construir el equilibrio entre ambas cuestiones, los requerimientos normativos y las necesidades políticas, y entusiasmar en la práctica a todos los actores involucrados del proceso de gestión de las políticas, sean o no militantes de BDP. Según una entrevistada "Teníamos voluntad de resolver los problemas, de hacernos cargo, no dejábamos a nadie tirado. Trabajábamos todo el día, y, además, los fines de semana. Entusiasmábamos a los de planta y demás

\footnotetext{
10 Según la información recolectada en el trabajo de campo, la subsecretaría contaba con alrededor de 182 trabajadores en todas sus dependencias, de los cuales el 60\% aproximadamente eran militantes de BDP, y el resto trabajadores de planta transitoria o permanente del Ministerio.
} 
trabajadores. Con dos pesos hacíamos políticas con fuerte impacto mediático" (Entrevista 13).

De la mano con esto, apareció otra limitación de la relación con los trabajadores de planta, el "secretismo". Es decir, la acusación de que BDP decidía unilateralmente y sin consultar cuestiones fundamentales de la gestión de los programas. La comunicación con los trabajadores, en este sentido, fue encarada como estratégica para evitar estas acusaciones y para evitar la circulación de comentarios o "chismes" que pudieran complicar la gestión cotidiana de la subsecretaría. Desde un primer momento, BDP se propuso construir una relación constructiva con los trabajadores de planta, al punto de que "se realizaba un trabajo político con ellos para sumarlos a la organización" (Entrevista 6).

\footnotetext{
"Según documentos públicos del Ministerio y las entrevistas realizadas, se puede pensar en un estilo de gestión donde el compromiso social y la gestión pública como forma de resolución de los problemas, se combinan en la idea de técnico-social. Este aspecto fue desarrollado con mayor profundidad en la tesis de maestría de la autora.

12 Perelmiter (2012), analiza en profundidad como se reconfiguró el rol del trabajador social y de su expertise en la micro política del Ministerio de Desarrollo social de la nación. El intento por reconstruir el vínculo entre el Estado y los sectores populares a partir de 2003, tiño las prácticas de los agentes estatales, que Perelmiter (2012) sintetiza en los conceptos de sensibilidad y territorialidad. Sensibilidad en tanto la personalización de la asistencia y el compromiso emocional desplegado por los trabajadores sociales, y territorialidad en tanto que el Estado "baja al territorio". Este "saber asistir" que los trabajadores sociales despliegan desde las agencias estatales del Ministerio, se condensa
}

\section{MILITAR, DISPUTAR Y REPRESENTAR AL ES- TADO}

En primer lugar, debemos señalar que el estilo de gestión de BDP no se puede entender sino en el marco del estilo de gestión del Ministerio ${ }^{11}$ durante el período analizado, ya que fue condición de posibilidad para la emergencia de esta experiencia ${ }^{12}$. Las prácticas y el saber hacer de BDP en los espacios analizados los denominamos "estilo de gestión militante”, cuya principal tecnología se puede sintetizar en la idea de "protagonismo" de sus principales actores, los militantes de BDP, cristalizado en la consigna "militar $y$ disputar el Estado". A este estilo de gestión subyace el supuesto de que las acciones de los actores políticos como según la autora en una serie de tecnologías de aproximación que intervienen en la práctica cotidiana del Estado. Un nuevo entramado institucional con fuerte presencia territorial, a través de los CIC, los centros de referencia y los diferentes programas, la modificación en la estructura y en la dinámica de trabajo con la incorporación y revalorización de los trabajadores sociales por sobre los técnicos de la etapa anterior; junto con los dispositivos de asistencia directa, fueron las herramientas utilizadas para redefinir la relación del Ministerio con los sectores populares. A su vez, estas estrategias de territorialización y sensibilización en la atención de demandas, dieron como resultado la emergencia de lo que Perelmiter denomina ethos militante (2012: 143), sensible a la atención de los sectores vulnerables, en oposición al ethos tecnocrático de las burocracias asistenciales. Es claro, en este trabajo, como los aspectos subjetivos de la práctica de los agentes estatales, como el compromiso y la sensibilidad, adquieren un lugar relevante en el sentido de la acción estatal. 
BDP deben ser comprendidas en relación a sus experiencias históricas y a sus marcos interpretativos desde donde construyen sus estrategias y tácticas cotidianas y a largo plazo. En esta línea consideramos que:

La forma en que las estructuras sociales condicionan la racionalidad de las acciones queda en evidencia al observar el modo en que la experiencia construye la "caja de herramientas" de estrategias, interpretaciones, y tácticas que son organizadas de acuerdo con lógicas relacionales (...) La experiencia conforma las categorías y lógicas empleadas por los actores al otorgar sentido a eventos presentes y posibles cambios futuros (Beltrán $\mathrm{y}$ Strauss, 2012, pp. 346-347).

El estilo de gestión estatal de BDP recupera sus experiencias y saberes, sus prácticas y las tecnologías de gestión utilizadas durante su participación territorial como vimos en el apartado anterior. Este estilo se caracterizó por presentar una mayor gravitación de los aspectos subjetivos por sobre los aspectos técnicos implicados en el proceso de implementación de una política estatal. La voluntad por "gestionar y de abrir el Estado y sus recursos", la capacidad individual de cada militante, y el compromiso para con los vecinos como objetivos políticos que guiaban la racionalidad de las prácticas de BDP en el Estado nacional, aparecieron como vimos en el apartado anterior, como elementos diferenciadores de este estilo.

A su vez, la propia experiencia previa de la organización introdujo elementos novedosos en la implementación de programas sociales. Perelmiter (2012) sostiene que el ingreso de las organizaciones de desocupados al Ministerio se apoyó en el reconocimiento por parte del Estado de las tareas sociales llevadas a cabo por estos actores durante la década anterior, y en la asignación de una misión específica como la relación entre el centro, el Estado y la periferia, el territorio. Este reconocimiento se cristaliza en la idea de que las organizaciones "subieron" al Estado, y desde ahí, su misión era "bajar al territorio". En ese "subir y bajar", las organizaciones de desocupados debían disputar la mediación de los sectores populares con otros agentes estatales, los burócratas de escritorio y los políticos locales. En esta resignificación de la mediación de los sectores populares, las organizaciones de desocupados debían "traducir su actividad política a un nuevo lenguaje" (Perelmiter, 2012, p.448), el saber burocrático. Conocer y dominar las prácticas de las técnicas administrativas 
fue un desafío que atravesó la experiencia de participación de BDP en la gestión estatal. $\mathrm{Y}$ en este sentido, Perelmiter señala una cuestión que apareció frecuentemente en el trabajo de campo, y que tiene que ver con la dimensión subjetiva y la dimensión técnica de la práctica de las organizaciones de desocupados en el Estado. En virtud de ello, el Estado también transforma a los militantes ya que deben abandonar su enraizamiento a un territorio particular, para asumir la universalización de lo local. Y por otro lado, los transforman en cuanto a que representar al Estado en el territorio implica recibir y asumir y canalizar una cantidad de demandas sociales, tensionando su capacidad de acción.

En este marco, y vinculado a la implementación de las iniciativas analizadas, aparece la aspiración de BDP a "representar al Estado nacional". Y aparece una consigna que sintetiza y expresa claramente esta cuestión: "militar el Estado" (Perelmiter, 2010; Gradin, 2012, 2014, 2016; Lozano; 2014; Vázquez, 2014), es decir "hacerse cargo de su representación y disputar su conducción política con otros actores". Como representantes del Estado los militantes tuvieron "llegada" a instituciones, organizaciones y vecinos con los que carecían de relación en tanto miembros de BDP. El "paraguas" del Estado nacional les abrió posibilidades de alianzas y relaciones que como organización social tenían vedadas. "Organizar" significaba establecer contacto con una cantidad grande de organizaciones e instituciones, siempre en el marco de un mínimo acuerdo político, para ser parte de la solución de sus propios problemas. Se sostenía una perspectiva comunitarista porque "se priorizaba el territorio", los "vecinos" y la relación con las organizaciones de la sociedad civil que componían el entramado social en el cual BDP se insertaba. Para esto era necesario "convocar", "promover el protagonismo popular", "organizar para representar". La consigna de "Militar el Estado" remitió claramente a la necesidad de reconstruir el entramado institucional, y la autoridad del mismo, de cara a la sociedad civil, cuestión que BDP asumió como propia a partir de su participación en el espacio político kirchnerista. Esto permitió a la organización potenciar su capacidad de relacionarse con otros actores, así como aumentar cuantitativa y cualitativamente su potencialidad de movilización y de referencia territorial.

De lo anterior se puede señalar que de la experiencia de participación debe BDP en el Ministerio, el estilo de gestión se caracterizó por la racionalidad política basada en la militancia en tanto tecnología legítima de gestión 
estatal (Gómez, 2010). La politización de la gestión, en tanto "militar, disputar y representar al Estado", fue una característica de la práctica cotidiana de BDP en la gestión del Ministerio que se desarrolla en este artículo. En consecuencia, se coincide con Vázquez (2014) que esta cuestión significó, por un lado, una legitimación de los capitales militantes de las organizaciones, y por otro, un proceso de reconversión de dichos saberes a partir de su participación en el Estado. El Estado, se constituyó así, en un espacio, entre otros, de militancia. $\mathrm{Y}$ en este escenario de militancia, la autora señala dos elementos que se vuelven importantes en la relación con la gestión. Las tareas burocráticas administrativas como "sacar los expedientes", se volvieron una tarea heroica y altruista, a la vez que las "buenas" causas defendidas fueron las que les otorgaron a los militantes la legitimidad de sus prácticas y experiencias ${ }^{13}$.

\section{REFLEXIONES FINALES}

La introducción del militante social como agente de gestión de los programas se contrapuso a la figura del "técnico" y al "burócrata" con que se asociaba la gestión estatal. Este nuevo agente combinaba la racionalidad po-

13 "Sacar los expedientes" remite al seguimiento y gestión de los expedientes administrativos lítica como lógica de acción, con tecnologías y formatos de intervención social de la tradición de los movimientos sociales. La imagen del militante como el agente que gestiona soluciones claramente depende de su voluntad, compromiso y capacidad para movilizar recursos, es decir de la capacidad política más que de las cuestiones legales administrativas. Su falta de experiencia en la gestión pública, así como la ausencia de técnicos o profesionales dentro de sus militantes, y su ímpetu por "resolver" las problemáticas sociales, le implicaron muchas veces no observar las normas legales, y accionar desde los márgenes de este esquema. Esta falta de observancias de las reglas formales, puso en diferentes oportunidades en riesgo el normal desarrollo de la gestión de los programas aquí analizados. De la experiencia realizada por BDP podemos decir que, a partir de ésta, se construyó una síntesis entre la lógica política presente en sus prácticas de gestión y la lógica burocrática administrativa propia del Estado.

Ahora bien, esta síntesis se inscribió en la organización y el comunitarismo como instrumentos claves que aportó BDP al hacer cotidiano del Estado. Este protagonismo como lei motiv del

que se requería para ejecutar los recursos económicos de los programas. 
trabajo estatal fue su principal característica que se pueden observar en las experiencias aquí desarrolladas. La incorporación de este tipo de organizaciones a la gestión pública trajo aparejado tensiones y transformaciones al interior de la administración pública, incorporando nuevos saberes y experiencias, así como una nueva correlación de fuerzas. Todo esto se expresó y se fue sintetizando parcialmente en el estilo de gestión estatal de BDP, construido a partir de la experiencia y de la adaptación de dicha organización a las normas y procedimientos requeridos para la gestión pública de recursos materiales, financieros o simbólicos.

Claro está, a manera de epílogo, que los resultados de esta experiencia son difíciles de medir. La renuncia de BDP a sus espacios de gestión y a su participación en el Ministerio, abortaron prematuramente la experiencia que duro tan sólo 5 años. La potencialidad de la participación política de BDP en el Estado nacional durante el periodo estudiado estuvo vinculada a su capacidad constructiva de mediaciones entre el Estado y la sociedad, más que en su capacidad de impactar en la transformación estructural de la sociedad argentina. En el proceso de ampliación del Estado encarado por el Kirchnerismo para resolver la crisis de 2001-2002, la participación política de las organizaciones de desocupados en la implementación de políticas estatales fue estratégica para suturar la crisis de representación política que atravesaba al país, y su estilo de gestión militante fue un aporte sustantivo a esta cuestión.

\section{REFERENCIAS BIBLIOGRÁFICAS}

Beltrán, G. J. y Strauss, L. (2012) "Expertos y dinámicas organizacionales: racionalidad limitada y consecuencias no buscadas en la Argentina de los noventa". En Morresi, Sergio y Vommaro, G. (eds.), Saber lo que se hace. Expertos y política en Argentina. Buenos Aires: Prometeo/UNG, 2012: 339 - 375.

Dallorso, N. (2013) "Alcances del concepto de dispositivo para el estudio de políticas sociales" Página 179 -202, En Debandi, N. et al. (eds.) Nuevos Horizontes de la investigación social: Artículos seleccionados de las VI Jornadas de Jóvenes Investigadores del Instituto Gino Germani. Ciudad Autónoma de Buenos Aires, CLACSO - IIGG, 2013.

Gómez, M. (2010) "Acerca del protagonismo político y la participación estatal de los movimientos sociales populares: juicio al para- 
digma normal de análisis" en Masetti, A., Villanueva, E. y Gómez, M. (eds.) Movilizaciones, protesta e identidades políticas en la Argentina del Bicentenario. Buenos Aires, Ed. Nueva Trilce. 2010: 65 - 95.

Gradin, A. (2016) "La participación política de las organizaciones de desocupados en el Estado nacional durante el periodo 2003 2009. La experiencia del Movimiento Barrios de pie: sus límites y potencialidades", Tesis de Doctorado, Doctorado en Ciencias Sociales, Facultad de Ciencias Sociales, Universidad de Buenos Aires.

Gradin, A. (2014). Estado y mediaciones sociales: El estilo de gestión del Movimiento de Desocupados Barrios de Pie en el territorio (Argentina 2002-2011). EstudiosCentro de Estudios Avanzados. Universidad Nacional de Córdoba, $N^{\circ}$ 32, 2014: 49-78.

Gradin, A (2013) El proceso de integración política de los Movimientos de Desocupados a la gestión del Estado en la Argentina Kirchnerista. Un análisis del estilo de gestión de los Programas Promotores Territoriales para el cambio Social y de Fortalecimiento y Participación de las Mujeres Juana
Azurduy durante el periodo 2003 - 2009" Tesis de Maestría en Políticas Públicas para el Desarrollo con Inclusión Social, FLACSO Argentina. Repositorio FLACSO ANDES. Disponible en http://repositorio.flacsoandes.edu.ec/bitstream/10469/5493 /1/TFLACSO-2013AG.pdf

Gradin, A. (2012) "Militar el Estado: las prácticas de gestión del Movimiento Barrios de Pie en el Programa de Promotores para el cambio social durante el periodo 2005 - 2008" En Revista Perspectivas de Políticas Públicas $N^{\circ} 3$ julio - diciembre 2012: 98 - 125.

Gradin, A. y Tiranti, S. (2012) “Del Programa Trabajar al Programa de ingreso social con trabajo "Argentina Trabaja": los nuevos dispositivos de gestión y su impacto en el gobierno local." Ponencia presentada en XI Seminario Red Muni "Repensando la Agenda Local", Universidad Nacional Arturo Jauretche, 18 y 19 de noviembre de 2012.

Lozano, J. I (2013) “Transformar el Estado, militándolo. Reflexiones sobre una experiencia de organizaciones sociales en la gestión de gobierno en la Provincia de Buenos Aires", ponencia presentada 
en X Jornadas de Sociología "20 años de pensar y repensar la sociología. Nuevos desafíos académicos, científicos y políticos para el siglo XXI" FSOC- UBA. 1 al 6 de julio de 2013.

Mancini, M. (2011) "Régimen político, gestión estatal e implementación de políticas a nivel subnacional. El caso de Santiago del Estero, Argentina." Revista Enfoques: Ciencias políticas y Administración pública. Vol. IX, Núm. 15. diciembre de 2011, pp.115 -176.

Masetti A. (2011) "Las tres transformaciones de la política pública asistencial y su relación con las organizaciones sociopolíticas (2003 - 2009)" en Entramados y Perspectivas Revista de la carrera de Sociología. Volumen $1 \mathrm{~N}^{\circ} 1$, Enero - Julio 2011:9 - 36.

Massetti, A. (2010) "Limitaciones de los movimientos sociales en la construcción de un Estado progresista en Argentina". Argumentos, Revista de Crítica Social $\mathrm{N}^{\circ} 12$, 2010: 81 - 108 en www.revistasigg.sociales.uba.ar/index.php/argumentos/article/view/118/108.

Aceseado el 1 de junio de 2011.

Mazzuca, S. (2002) "¿Democratización o Burocratización? Inestabi- lidad del acceso al poder y estabilidad del ejercicio del poder en América Latina". En Araucaria Revista Iberoamericana de Filosofía, Política y Humanidades, Año 4, N - 7, Primer Semestre de 2002.

Perelmiter, L. (2012) Fronteras inestables y eficaces. El ingreso de organizaciones de desocupados a la burocracia asistencial del Estado. Argentina (2003-2008) en Estudios Sociológicos, Vol. 30, No. 89 (mayo-agosto, 2012), pp. 431458.

Perelmiter, L. (2010) "Militar el Estado. La incorporación de movimientos sociales de desocupados a la gestión de políticas sociales. Argentina (2003 - 2008)" Páginas 137 - 156, en Masetti, A., Villanueva, E. y Gómez, M. (eds.) Movilizaciones, protesta e identidades políticas en la Argentina del $\mathrm{Bi}$ centenario. Buenos Aires, Ed. Nueva Trilce.

Schavelzon, S. (2007) "El gestionismo y el politicismo en el Estado: el caso de dos estilos de gobierno en la comisión municipal de la Vivienda de la ciudad de Buenos Aires" en Revista Campos $N^{\circ} 8$ (2). Brasil, 2007: 41 - 63.

Tiranti, S. (2013) "Nación y Munici- 
pios: nuevos dispositivos de gestión en el territorio. Una mirada desde el conurbano bonaerense 2004 - 2012" Tesis de Maestría en Políticas Públicas para el Desarrollo con Inclusión Social FLACSO sede académica Argentina. Disponible en http://http//www.flacsoandes.org/dspace/bitstream/10469/ 5917/2/TFLACSO-2013SDT.pdf

Vázquez, M. (2014) «Militar la gestión»: una aproximación a las relaciones entre activismo y trabajo en el Estado a partir de las gestiones de gobierno de Cristina Fernández de Kirchner en Argentina" en Revista Apuntes 74, primer semestre, 2014: 71 -102.

Zaremberg, G. (2004) "Alpargatas y libro: estilos de gestión, género y política social en Argentina $y$ Chile" Santiago de Chile, CEPAL Serie Políticas Sociales N ${ }^{\circ} 90$.

\section{PARA CITAR ESTE ARTÍCULO:}

Gradin, A. (2017). Protagonismo y militancia: el estilo de gestión del movimiento barrios de pie en el Ministerio Nacional de Desarrollo Social, Argentina 2004-2009. Collectivus, Revista de Ciencias Sociales, 4(2), 141-161.

DOI: http://dx.doi.org/10.15648/Coll.2.2017.8

Recibido: 25/02/2017 Aprobado: 15/05/2017 